\title{
Adalimumab Reduces Extraintestinal Manifestations in Patients with Crohn's Disease: A Pooled Analysis of 11 Clinical Studies
}

\author{
Edouard J. Louis • Walter Reinisch • David A. Schwartz • Robert Löfberg • Anne M. Robinson • \\ Sofie Berg · Anthony W. Wang · Jen-fue Maa · Bidan Huang $\cdot$ Brandee Pappalardo
}

Received: December 4, 2017 / Published online: March 7, 2018

(C) The Author(s) 2018. This article is an open access publication

\begin{abstract}
Introduction: Extraintestinal manifestations (EIMs) in patients with Crohn's disease (CD) are common and associated with additional morbidity. This study aimed to evaluate the effect of adalimumab therapy on EIM resolution and identify potential predictors of EIM resolution
\end{abstract}

Enhanced content To view enhanced content for this article go to https://doi.org/10.6084/m9.figshare. 5896612 .

Electronic supplementary material The online version of this article (https://doi.org/10.1007/s12325018-0678-0) contains supplementary material, which is available to authorized users.

E. J. Louis $(\bowtie)$

University Hospital CHU of Liège, Liège, Belgium

e-mail: edouard.louis@ulg.ac.be

W. Reinisch

McMaster University, Hamilton, ON, Canada

W. Reinisch

Medical University of Vienna, Vienna, Austria

D. A. Schwartz

Vanderbilt University, Nashville, TN, USA

R. Löfberg

Karolinska Institutet, Sophiahemmet, Stockholm, Sweden

A. M. Robinson - S. Berg · A. W. Wang · J. Maa .

B. Huang · B. Pappalardo

AbbVie Inc., North Chicago, IL, USA in adult and pediatric patients with moderate to severe CD.

Methods: EIM data were pooled from 11 induction, maintenance, and open-label extension studies of adalimumab. Resolution of EIMs was evaluated at approximately 6 months and 1 year. Median time to initial EIM resolution and first EIM recurrence (reflecting durable resolution) of any EIM and specific categories of EIMs (arthritis/arthralgia, ocular, cutaneous) were calculated. A Cox model was used to determine predictors of initial and durable EIM resolution.

Results: At baseline, 54\% (1137/2094) of patients receiving adalimumab and 51\% (297/ 586) receiving placebo had EIMs. EIM resolution occurred in a significantly greater proportion of adalimumab versus placebo patients at 6 months $(54 \%$ vs $31 \% ; P<.001)$ and 1 year (60\% vs $42 \% ; P=.008$ ). Median time to initial resolution of any EIM, arthritis/arthralgia, and cutaneous EIMs was significantly shorter in patients receiving adalimumab versus placebo. Durable resolution of any EIM and arthritis/ arthralgia was significantly longer for patients receiving adalimumab versus placebo. Clinically meaningful predictors of EIM resolution included adalimumab treatment, male sex, and moderate (versus severe) disease activity at baseline.

Conclusion: Adalimumab is effective for achieving initial and durable resolution of any EIM and, in particular, arthritis/arthralgia in 
patients with moderate to severe $\mathrm{CD}$. Predictors of EIM resolution included adalimumab treatment and moderate disease severity.

Funding: AbbVie.

Keywords: Adalimumab; Arthralgia; Arthritis; CDAI; Crohn's disease; Extraintestinal manifestation; Gastroenterology; Inflammatory bowel disease

\section{INTRODUCTION}

Although Crohn's disease (CD) is a chronic, recurrent inflammatory disease of the digestive tract, the impact of CD can extend beyond this primary location. Extraintestinal manifestations (EIMs), such as arthritis and arthralgia, are present in up to $70 \%$ of patients with CD and are associated with morbidity and impaired quality of life [1-6]. Although the pathogenesis of EIMs remains unclear, some manifestations appear to be related to the pathophysiology of inflammatory bowel disease (IBD) [5, 7].

In $\mathrm{CD}$, the risk of developing EIMs is increased in patients who smoke, have longer disease duration, and are younger at diagnosis $[3,8]$. The presence of one EIM may confer a higher likelihood of developing other EIMs [7]. Although EIMs that parallel IBD activity may resolve with treatment of intestinal inflammation, EIMs that are independent of intestinal disease activity will likely require treatment with additional systemic therapies $[6,9,10]$.

Adalimumab, a recombinant human monoclonal antibody targeting tumor necrosis factor (TNF), is effective in the induction and maintenance of remission of moderate to severe CD in adult and pediatric patients [11-14]. The objectives of this post hoc analysis of pooled study data from adult and pediatric patients with moderate to severe $C D$ were to evaluate the effect of adalimumab therapy on initial and durable EIM resolution and to describe potential predictors of initial resolution and first recurrence of EIMs.

\section{METHODS}

\section{Study Designs and Patients}

Data related to EIMs were pooled from 11 induction, maintenance, and open-label extension studies in adult and pediatric patients with moderate to severe CD (Supplemental Fig. S1). The methods and primary results of the CD trials were previously reported (Supplemental Table S1). This article does not contain any new studies with human or animal subjects performed by any of the authors [11-22]. Adult patients were enrolled in one of the following studies: three double-blind, randomized, placebo-controlled induction studies (Clinical assessment of Adalimumab Safety and efficacy Studied as Induction therapy in Crohn's disease [CLASSIC-I; NCT00055523], Gauging Adalimumab efficacy in Infliximab Nonresponders [GAIN; NCT00105300], and M04-729 Japan [NCT00445939]), including follow-up in their 52 -week maintenance or extension studies (CLASSIC-II [NCT00055497], Additional longterm Dosing with HUMIRA to Evaluate sustained Remission and Efficacy in CD [ADHERE; NCT00195715], and M06-837 [NCT00445432], respectively); two double-blind, randomized, placebo-controlled, 52-week (extend the safety and efficacy of adalimumab through endoscopic healing [EXTEND; NCT00348283]) or 56-week (Crohn's trial of the fully Human antibody Adalimumab for Remission Maintenance [CHARM; NCT00077779]) induction and maintenance studies; and one 20-week, openlabel study (Crohn's Treatment with Adalimumab: Patient Response to a Safety and Efficacy Study [CARE; NCT00409617]). Pediatric patients were enrolled in one randomized, double-blind, 52-week pediatric study (IMAgINE 1; NCT00409682) and its open-label extension (IMAgINE 2; NCT00686374); only patients at least 13 years old who completed the Crohn's Disease Activity Index (CDAI) were included in this analysis.

Patients included in this analysis had baseline and postbaseline EIM data. The adalimumab group included all patients randomized to adalimumab, including those who received 
open-label rescue therapy after blinded adalimumab treatment or open-label adalimumab in non-randomized trials. The placebo group included patients who were randomized to placebo in the double-blind trials. Patients who received placebo and were subsequently rerandomized to blinded adalimumab or received open-label adalimumab at any point were censored from that point forward.

\section{EIM Assessment}

In 10 studies, assessment of the presence or absence of EIMs was based on question 4 of the CDAI, which evaluated these EIM categories: arthritis or arthralgia, ocular complications (iritis or uveitis), and cutaneous complications (erythema nodosum, pyoderma gangrenosum, or aphthous stomatitis). In the CARE study, the presence of EIMs was evaluated by the investigator on a separate form [18].

Resolution of EIMs was evaluated in a pooled analysis of all studies at the time points closest to 6 months (weeks 20-26) and 1 year (weeks 52-56). Because CARE was a 20-week study, it was included in the 6-month analysis but not the 1-year analysis. Corticosteroid-free EIM resolution was evaluated at 6 months and 1 year in patients with EIMs at baseline who also received corticosteroids at baseline. Time to initial EIM resolution and time to first EIM recurrence (reflecting durable resolution) were calculated for any EIM and for specific categories of EIMs (arthritis/arthralgia, ocular, cutaneous). Recurrence was defined by the presence of the EIM after initial resolution.

To identify potential predictors of initial EIM resolution and first EIM recurrence, these baseline characteristics were evaluated: sex, age, disease duration, involved intestinal area, disease activity (CDAI or Harvey-Bradshaw Index), albumin (g/L), C-reactive protein $(\mathrm{mg} / \mathrm{dL})$, thiopurine use, corticosteroid use, prior antiTNF exposure, and smoking status.

\section{Statistical Analyses}

A $t$ test (continuous predictors) or Chi-square test (categorical predictors) was used to evaluate potential differences in baseline characteristics between groups for (1) patients with versus without EIMs at baseline and (2) placebo versus adalimumab groups in patients with EIMs at baseline. Among patients with EIMs and corticosteroid use at baseline, corticosteroid-free EIM resolution (EIM resolution in patients who also discontinued corticosteroids) was evaluated. Because of the disproportionately small number of patients in the placebo group relative to the adalimumab group, between-group comparisons were descriptive only and $P$ values were not provided. Data are reported as observed.

Median time to initial EIM resolution and first EIM recurrence following resolution were evaluated via Kaplan-Meier survival curves with the log-rank test for between-group comparisons; median time to first recurrence of cutaneous and ocular EIMs could not be estimated because of low event numbers. A random-effect meta-analysis that used the study as a random effect in a Cox model determined the predictors of initial and durable EIM resolution in patients with any EIM or with arthritis/arthralgia.

\section{RESULTS}

\section{Patients}

The contribution of patients from each of the included studies is outlined in Supplemental Table S1 and Supplemental Fig. S1. A total of $54 \%(1434 / 2680)$ of patients had EIMs at baseline $(51 \%$ of placebo patients [297/586] and $54 \%$ of adalimumab patients [1137/2094]). Arthritis/arthralgia was present in $51 \%$ of patients (48\% of placebo patients [282/586] and $52 \%$ of adalimumab patients [1082/2094]) and represented $95 \%$ of all EIMs reported in these studies (Table 1). Only a small proportion of patients had ocular (1\%; 33/2680) or cutaneous (6\%; 157/2680) EIMs. Nearly all baseline characteristics were significantly different for patients with EIMs versus those without EIMs; patients with EIMs were more likely to be women, smokers, slightly older, with longer disease duration, and more severe disease (Table 1). Patients with EIMs were also less likely to use thiopurines at baseline and more likely to 
Table 1 Patient demographics and baseline disease characteristics

\begin{tabular}{|c|c|c|c|c|c|c|}
\hline \multirow[t]{2}{*}{ Characteristic } & \multicolumn{3}{|c|}{ Overall ITT population } & \multicolumn{3}{|c|}{ Patients with EIMs at baseline } \\
\hline & $\begin{array}{l}\text { EIMs at } \\
\text { baseline } \\
n=1434\end{array}$ & $\begin{array}{l}\text { No EIMs at } \\
\text { baseline } \\
n=1246\end{array}$ & $P$ value & $\begin{array}{l}\text { Placebo } \\
n=297\end{array}$ & $\begin{array}{l}\text { Adalimumab } \\
n=1137\end{array}$ & $P$ value \\
\hline Age, years, mean $(\mathrm{SD})$ & $37.8(12.1)$ & $33.0(11.7)$ & $<.001$ & $\begin{array}{l}38.9 \\
\quad(11.9)\end{array}$ & $37.5(12.2)$ & .090 \\
\hline Female, $n(\%)$ & $943(65.8)$ & $646(51.8)$ & $<.001$ & $\begin{array}{l}196 \\
(66.0)\end{array}$ & $747(65.7)$ & .924 \\
\hline $\begin{array}{l}\text { Disease duration, years, mean } \\
\text { (SD) }\end{array}$ & $\begin{array}{l}10.4(8.6) \\
n=1433\end{array}$ & $9.1(7.6)$ & $<.001$ & $\begin{array}{l}11.0(8.8) \\
n=296\end{array}$ & $10.3(8.5)$ & .202 \\
\hline Disease activity $^{\mathrm{a}}$ & & & .018 & & & .001 \\
\hline Moderate, $n(\%)$ & $835(58.2)$ & $782(62.8)$ & & $\begin{array}{l}149 \\
\quad(50.2)\end{array}$ & $686(60.3)$ & \\
\hline Severe, $n(\%)$ & $598(41.7)$ & $464(37.2)$ & & $\begin{array}{l}148 \\
\quad(49.8)\end{array}$ & $450(39.6)$ & \\
\hline Albumin, g/L, mean (SD) & $39.7(5.0)$ & $38.9(5.3)$ & $<.001$ & $39.6(4.8)$ & $39.7(5.0)$ & .701 \\
\hline \multirow[t]{2}{*}{$\mathrm{CRP}, \mathrm{mg} / \mathrm{dL}$, mean $(\mathrm{SD})$} & $1.9(2.7)$ & $2.4(4.3)$ & $<.001$ & $1.7(2.7)$ & $1.9(2.7)$ & .248 \\
\hline & $n=1421$ & $n=1227$ & & & $n=1124$ & \\
\hline \multicolumn{7}{|c|}{ Concomitant medication use at baseline, $n(\%)$} \\
\hline Thiopurine use (AZA/6-MP) & $554(38.6)$ & $548(44.0)$ & .005 & $\begin{array}{l}105 \\
\quad(35.4)\end{array}$ & $449(39.5)$ & .192 \\
\hline Corticosteroid use & $587(40.9)$ & $503(40.4)$ & .766 & $\begin{array}{l}117 \\
\quad(39.4)\end{array}$ & $470(41.3)$ & .544 \\
\hline Prior anti-TNF use & $736(51.3)$ & $592(47.5)$ & .049 & $\begin{array}{l}186 \\
(62.6)\end{array}$ & $550(48.4)$ & $<.001$ \\
\hline Current smoker, $n(\%)$ & $545(38.0)$ & $411(33.0)$ & .007 & $\begin{array}{l}107 \\
\quad(36.0)\end{array}$ & $438(38.5)$ & .430 \\
\hline \multicolumn{7}{|l|}{ EIMs at baseline $\mathrm{b}^{\mathrm{b}}$} \\
\hline Arthritis/arthralgia & $1364(95.1)$ & NA & & $\begin{array}{l}282 \\
\quad(94.9)\end{array}$ & $1082(95.2)$ & \\
\hline Ocular & $33(2.3)$ & NA & & $6(2.0)$ & $27(2.4)$ & \\
\hline
\end{tabular}


Table 1 continued

\begin{tabular}{|c|c|c|c|c|c|c|}
\hline \multirow[t]{2}{*}{ Characteristic } & \multicolumn{3}{|c|}{ Overall ITT population } & \multicolumn{3}{|c|}{ Patients with EIMs at baseline } \\
\hline & $\begin{array}{l}\text { EIMs at } \\
\text { baseline } \\
n=1434\end{array}$ & $\begin{array}{l}\text { No EIMs at } \\
\text { baseline } \\
n=1246\end{array}$ & $P$ value & $\begin{array}{l}\text { Placebo } \\
n=297\end{array}$ & $\begin{array}{l}\text { Adalimumab } \\
n=1137\end{array}$ & $P$ value \\
\hline Cutaneous & $157(10.9)$ & NA & & $23(7.7)$ & $134(11.8)$ & \\
\hline
\end{tabular}

Statistical comparison for continuous variables from $t$ test, and categorical variables from Chi-square test

6-MP 6-mercaptopurine, $A Z A$ azathioprine, CDAI Crohn's Disease Activity Index, CRP C-reactive protein, EIM extraintestinal manifestation, $H B I$ Harvey-Bradshaw Index, ITT intent to treat, $N A$ not applicable, TNF tumor necrosis factor

${ }^{a}$ Missing information for 1 placebo patient with EIMs. Disease activity based on the CDAI (moderate disease activity $=\mathrm{CDAI} \leq 300)$ or $\mathrm{HBI}(\mathrm{CARE}$ study; moderate disease activity $=\mathrm{HBI} \leq 16)$

b Patients could have $\geq 1$ type of EIM

have prior anti-TNF use. In contrast, among patients with EIMs at baseline, patient characteristics were generally similar between placebo and adalimumab groups with the exception that significantly more patients receiving placebo had severe disease activity (50\% vs $40 \%$; $P=.001)$ and prior anti-TNF use $(63 \%$ vs $48 \%$; $P<.001)$ versus patients receiving adalimumab (Table 1).

\section{Proportion of Patients with Resolution of EIMs}

Among patients with any EIM or arthritis/ arthralgia at baseline, resolution occurred in a significantly greater proportion of adalimumab

(a)

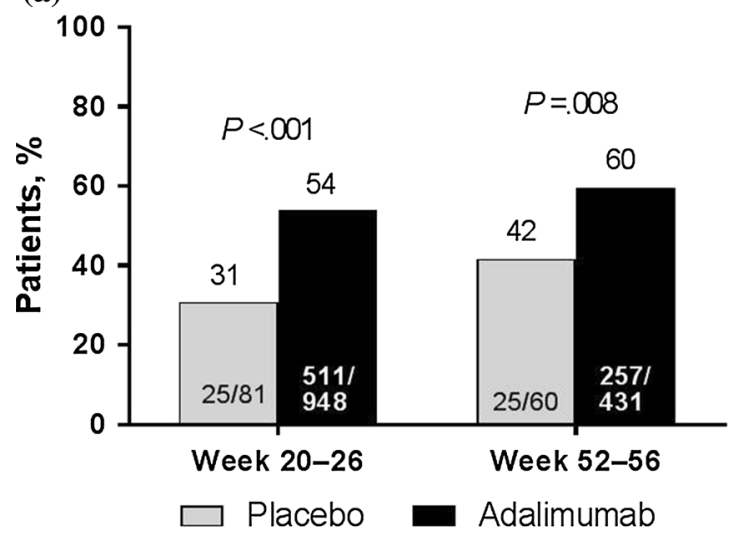

versus placebo patients at 6 months (both $P<.001$ ) and 1 year (both $P=.008$; Fig. $1 \mathrm{a}, \mathrm{b}$ ). All patients receiving adalimumab with ocular EIMs at baseline achieved resolution at 6 months (21/21) and 1 year (9/9) compared with $2 / 3$ and $2 / 2$ patients receiving placebo at the same time points, respectively. Resolution of cutaneous EIMs was achieved by 90\% (96/ $107)$ of patients receiving adalimumab at 6 months and 95\% (35/37) at 1 year versus $3 / 3$ and $2 / 3$ patients receiving placebo, respectively. A higher proportion of patients receiving adalimumab experienced corticosteroid-free EIM resolution at 6 months and 1 year compared with those receiving placebo; more than $60 \%$ of patients receiving adalimumab who were also taking corticosteroids at baseline had

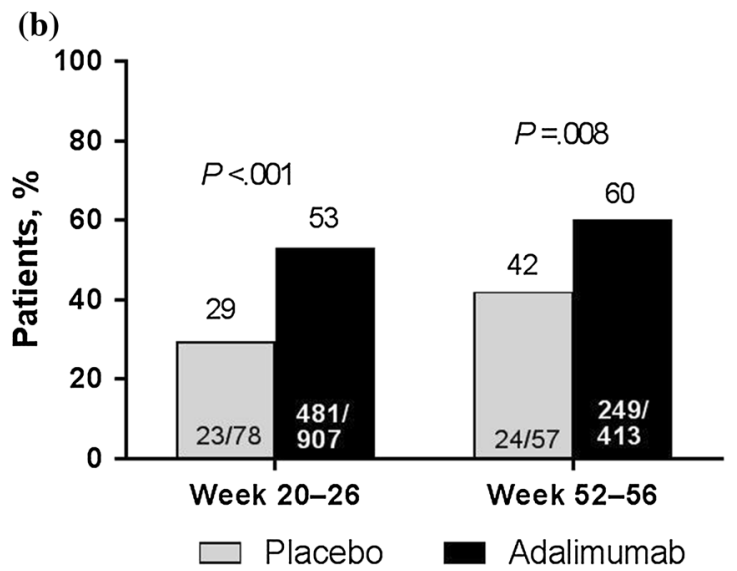

Fig. 1 Proportion of patients with resolution of $\mathbf{a}$ any EIM or $\mathbf{b}$ arthritis or arthralgia at 6 months (weeks 20-26) and 1 year (weeks 52-56). $P$ values for between-group comparison from Chi-square test. EIM extraintestinal manifestation 
corticosteroid-free EIM resolution at 1 year (Fig. 2).

\section{Time to Resolution of First EIM and Time to First EIM Recurrence}

Compared with patients receiving placebo, those receiving adalimumab had significantly shorter median time to resolution of any EIM and arthritis/arthralgia (both 43 vs 155 days; $P<.001)$ and cutaneous EIMs (15 vs 52 days; $P=.040$; Fig. 3a-c). Among the few patients with ocular EIMs, median time to resolution of first EIM was numerically shorter for the adalimumab group compared with the placebo group (19 vs 30 days; $P=.073$; Fig. $3 d$ ). For patients who achieved initial EIM resolution, median time to first EIM recurrence was significantly longer for the adalimumab group versus the placebo group for any EIM (137 vs 57 days; $P<.001)$ and for arthritis/arthralgia (141 vs 57 days; $P<.001$; Fig. $4 \mathrm{a}, \mathrm{b})$.

\section{Predictors of Initial EIM Resolution and First EIM Recurrence}

Although several baseline variables were found to be significant predictors of initial resolution of any EIM and arthritis/arthralgia, the variables that showed the greatest difference included adalimumab treatment and moderate (versus severe) disease activity (Fig. 5a). The strongest

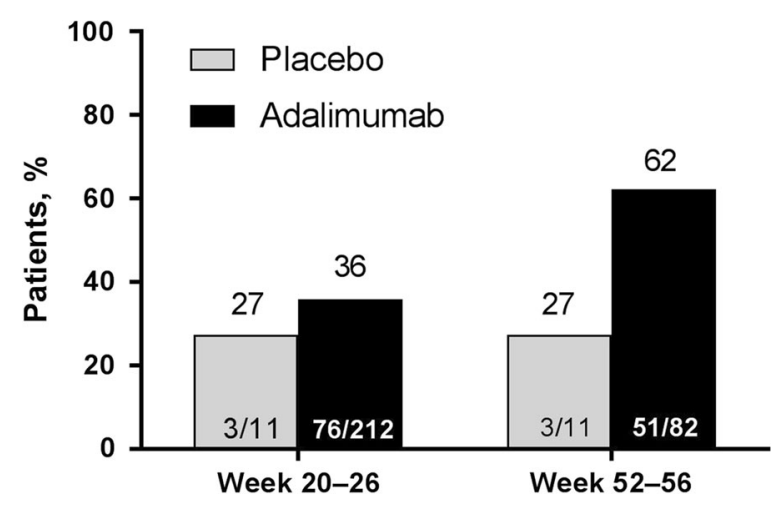

Fig. 2 Proportion of patients with corticosteroid-free extraintestinal manifestation resolution at 6 months (weeks 20-26) and 1 year (weeks 52-56) baseline predictors of EIM recurrence were placebo treatment and female sex for any EIM and placebo treatment for arthritis/arthralgia (Fig. 5b). There were no associations between initial EIM resolution or EIM recurrence with baseline smoking status or use of thiopurines, corticosteroids, or prior anti-TNFs. Furthermore, disease location was not a significant factor for EIM resolution. Predictors of resolution and recurrence of cutaneous and ocular EIMs could not be estimated because of low event numbers.

\section{DISCUSSION}

Arthritis and arthralgia represent the most common EIMs observed in patients with IBD $[7,8]$; joint symptoms are reported in up to $40 \%$ of patients $[23,24]$. This post hoc analysis of pooled data from 11 adalimumab clinical trials demonstrated that $54 \%$ of patients with moderate to severe $\mathrm{CD}$ had at least one EIM at baseline and significantly more patients receiving adalimumab achieved resolution of EIMs at 6 months and 1 year versus patients receiving placebo. Furthermore, arthritis/arthralgia was present in $51 \%$ of patients and represented 95\% of all EIMs reported in these studies. The contribution of arthritis/arthralgia to IBD morbidity is highlighted in that it is the only EIM included as a component of the IBD disability index [25]. Of note, most of the data were collected using question 4 of the CDAI, which does not distinguish between arthritis and arthralgia. To date, this is the largest study to report the effects of an anti-TNF agent on EIM resolution among patients with moderate to severe CD.

Although arthritis and arthralgia are the most common and likely to be the first presenting EIMs in CD [26], patients can also present with a variety of other manifestations. Erythema nodosum and pyoderma gangrenosum are among the most common cutaneous manifestations reported in $\mathrm{CD}$, with a prevalence of $1-13 \%$ [2, 26-28], although prevalence up to $20 \%$ has been reported in some studies $[8,29]$. The reported incidence of ocular EIMs shows an even greater variation between studies (4-29\%) [2, 5, 26]. In this pooled analysis of patients with moderate to severe $\mathrm{CD}$ in 
(a)

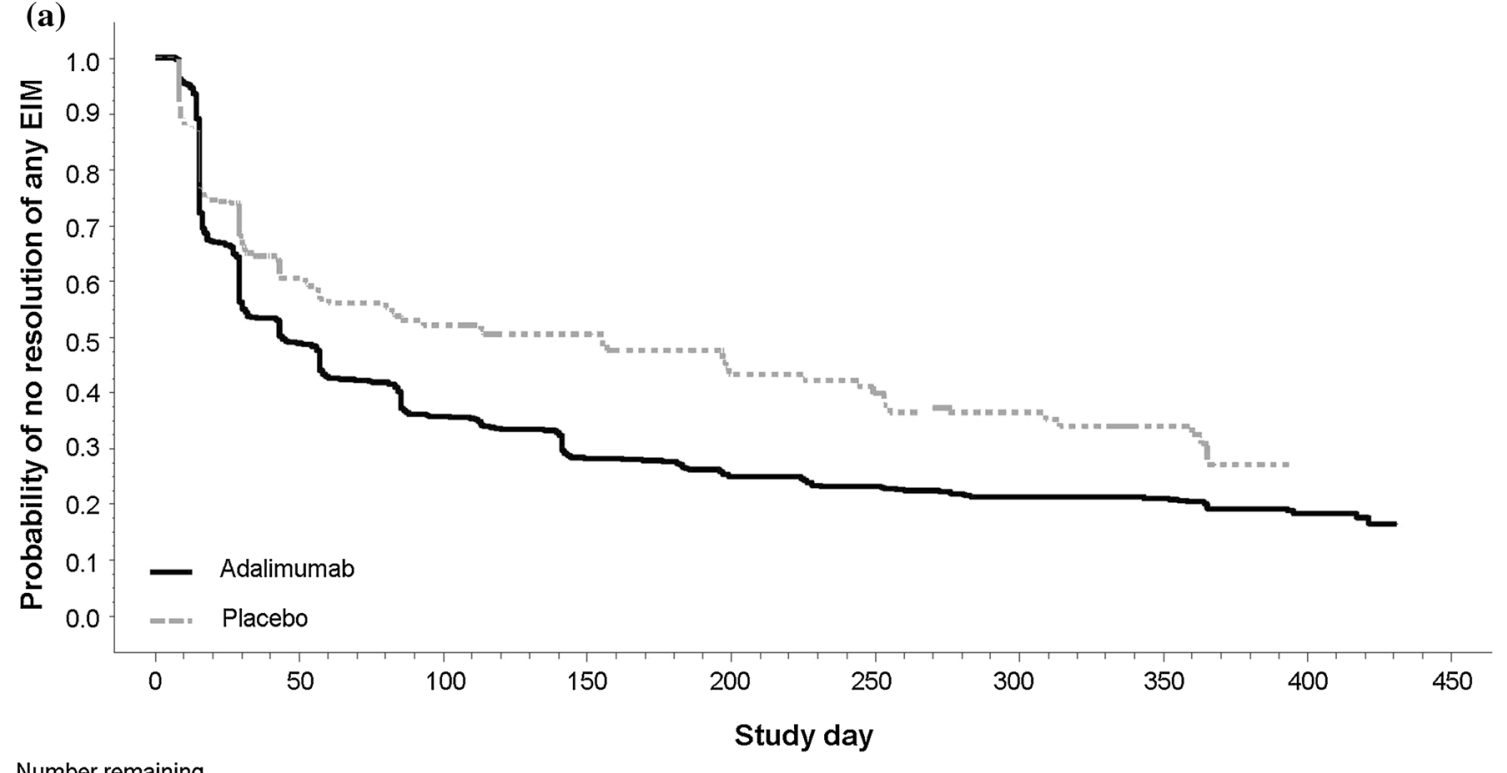

$\begin{array}{cccccccccc}\text { Number remaining } & & & & & \\ \text { ADA } & 1137 & 527 & 348 & 162 & 127 & 112 & 95 & 92 & 26 \\ \text { PBO } & 297 & 80 & 63 & 54 & 41 & 35 & 29 & 24 & 0\end{array}$

(b)

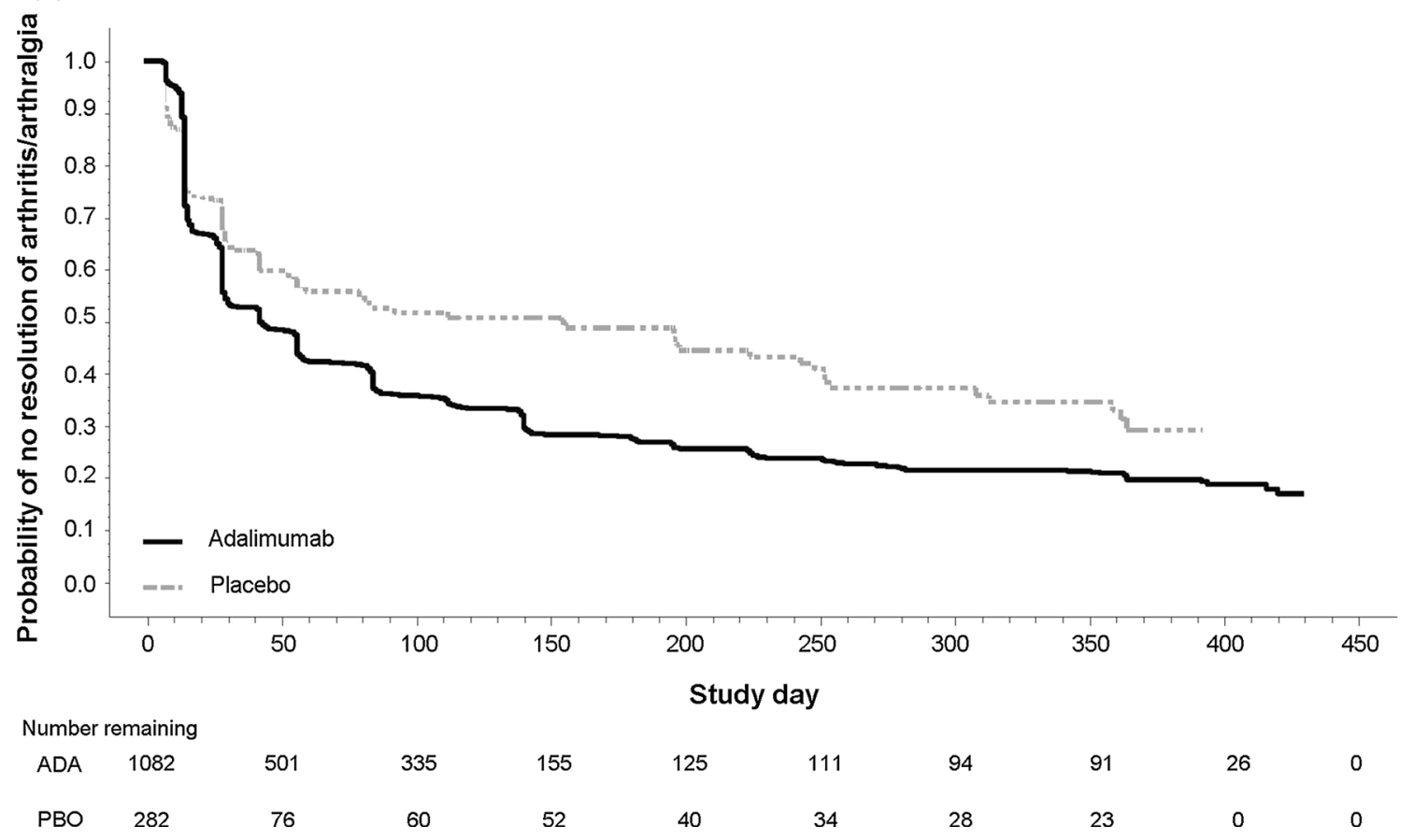

Fig. 3 Time to resolution of first EIM in patients with $\mathbf{a}$ any EIM, $\mathbf{b}$ arthritis or arthralgia, $\mathbf{c}$ cutaneous EIMs, and $\mathbf{d}$ ocular EIMs at baseline. $A D A$ adalimumab, EIM extraintestinal manifestation, $P B O$ placebo

adalimumab clinical trials, $6 \%$ of patients presented with cutaneous EIMs and $1 \%$ presented with ocular EIMs.
Recommended therapies for EIMs vary depending on disease activity and include corticosteroids, conventional immunosuppressants, non-steroidal anti-inflammatory drugs, 
(c)

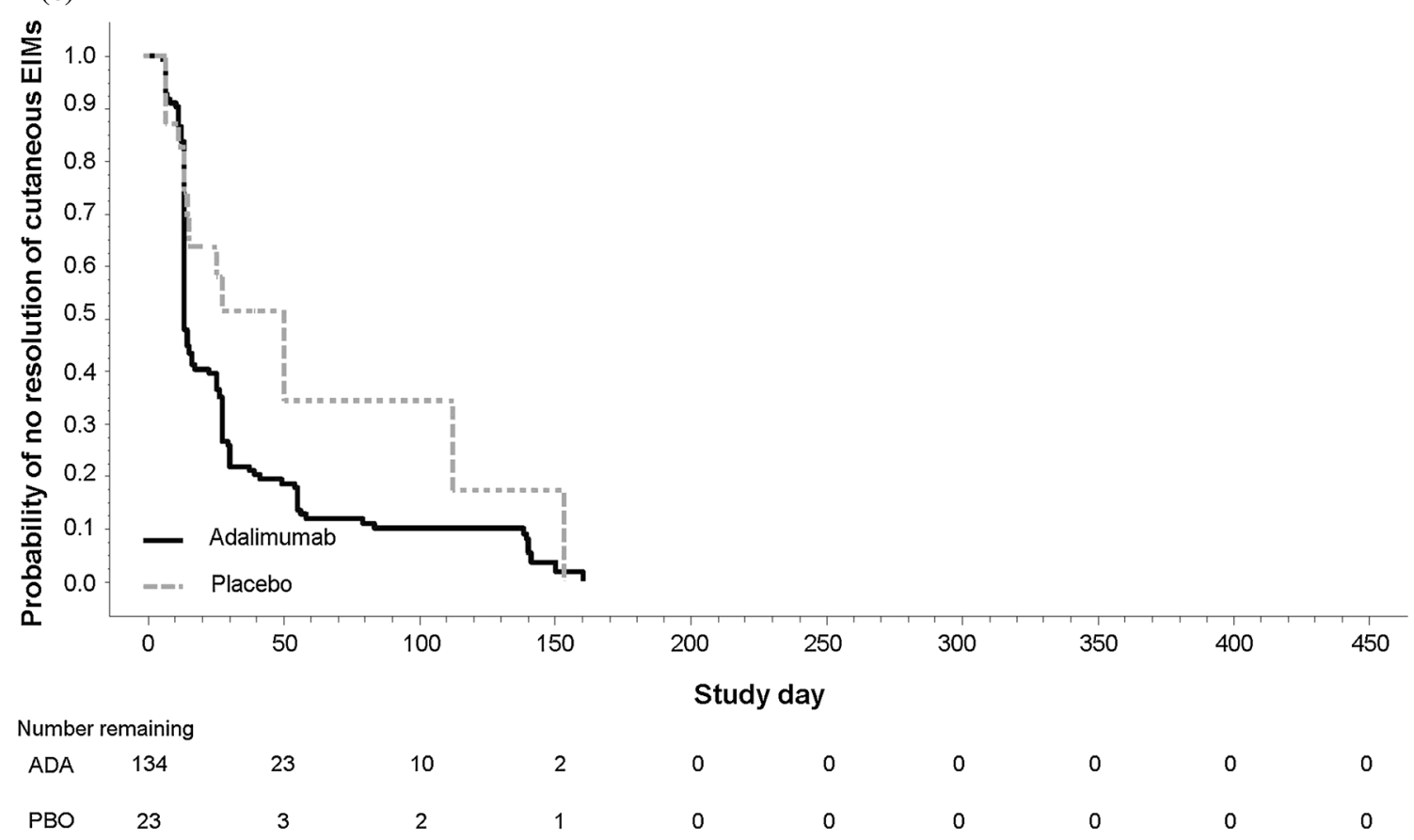

(d)

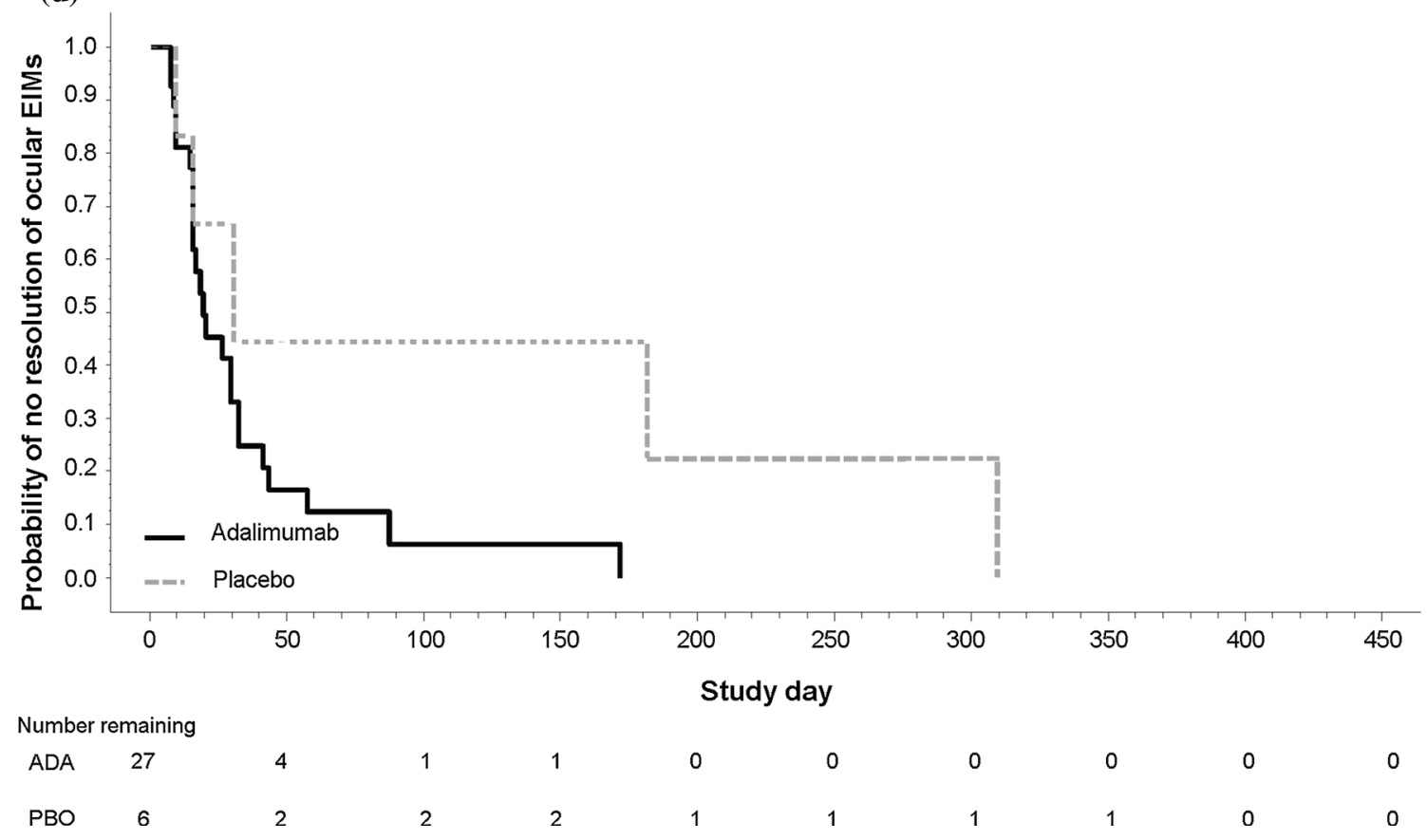

Fig. 3 continued

and anti-TNF agents [5]. The benefit of anti-TNF therapies in the management of EIMs is likely a result of underlying TNF- $\alpha$-dependent pathogenesis, which is common to both IBD and some comorbid immune-mediated inflammatory diseases affecting joints, skin, or eyes 

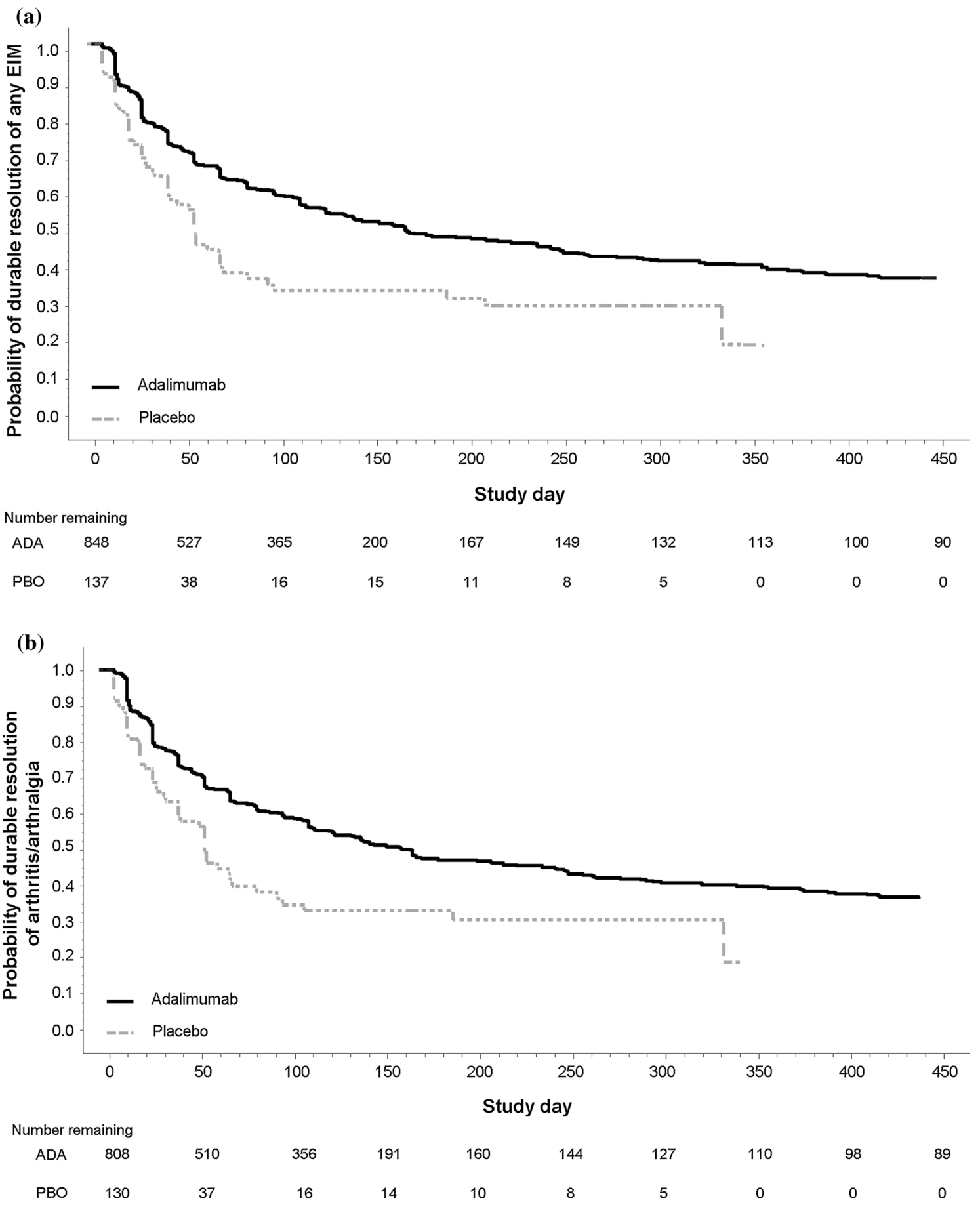

Fig. 4 Median time to first EIM recurrence (durable EIM resolution) of $\mathbf{a}$ any EIM and $\mathbf{b}$ arthritis or arthralgia following initial resolution. $A D A$ adalimumab, $E I M$ extraintestinal manifestation, $P B O$ placebo

$[5,9,30]$. It is unclear if biologic agents that do not target mediators of systemic inflammation will have the same impact on EIMs. A recent post hoc analysis of GEMINI 2 failed to show a statistically significant benefit of the gutselective agent vedolizumab over placebo for 
(a)

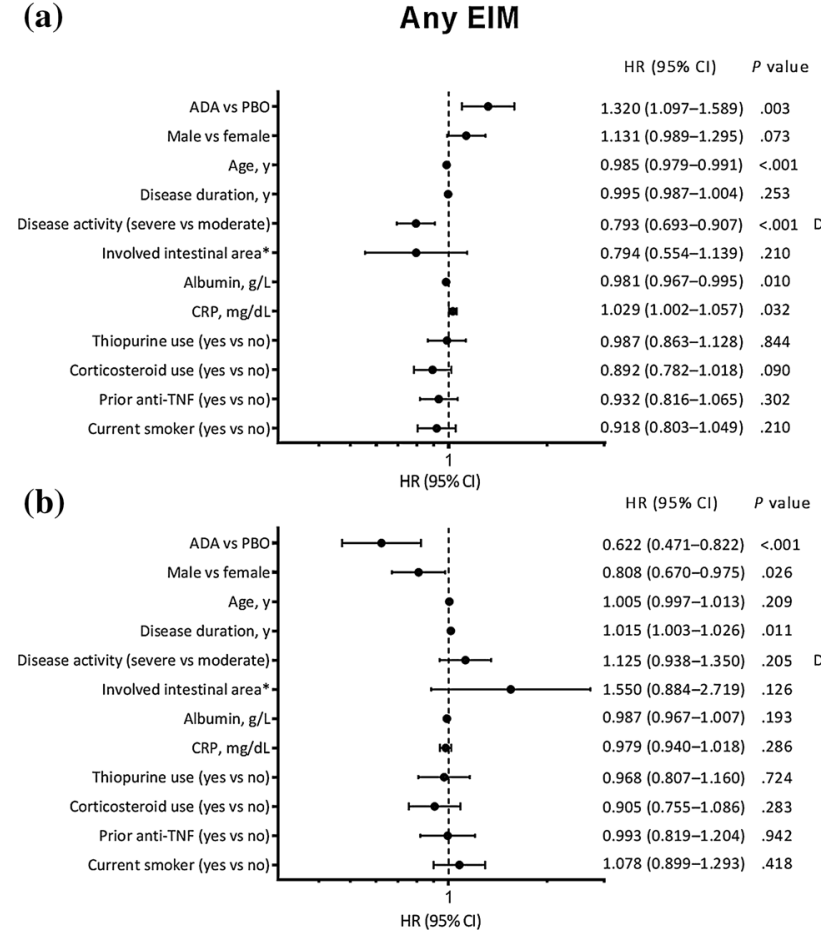

Fig. 5 Predictors of a EIM resolution and $\mathbf{b}$ first EIM recurrence. Hazard ratio (95\% CI) for time to first resolution or first recurrence. $P$ value from a Cox model. $A D A$ adalimumab, CRP C-reactive protein, EIM

the treatment of EIMs in patients with CD [31]. In contrast, systemic acting anti-TNF agents have been shown to successfully treat a variety of EIMs [32-38]. Furthermore, the first European evidence-based consensus on EIMs in IBD supports the use of anti-TNFs for the treatment of several EIMs, including peripheral arthritis, scleritis/uveitis, erythema nodosum, and pyoderma gangrenosum [5].

Data from this analysis support these recommendations. In our pooled analysis, more than $50 \%$ of patients receiving adalimumab achieved resolution of any EIM and arthritis/ arthralgia at 6 months and 1 year. Importantly, in contrast to patients receiving placebo, more patients receiving adalimumab were able to discontinue corticosteroids and still achieve EIM resolution. In addition, the adalimumab group had significantly shorter time to initial EIM resolution and longer time to first EIM recurrence compared with the placebo group. These findings are consistent with real-world
Arthritis/Arthralgia

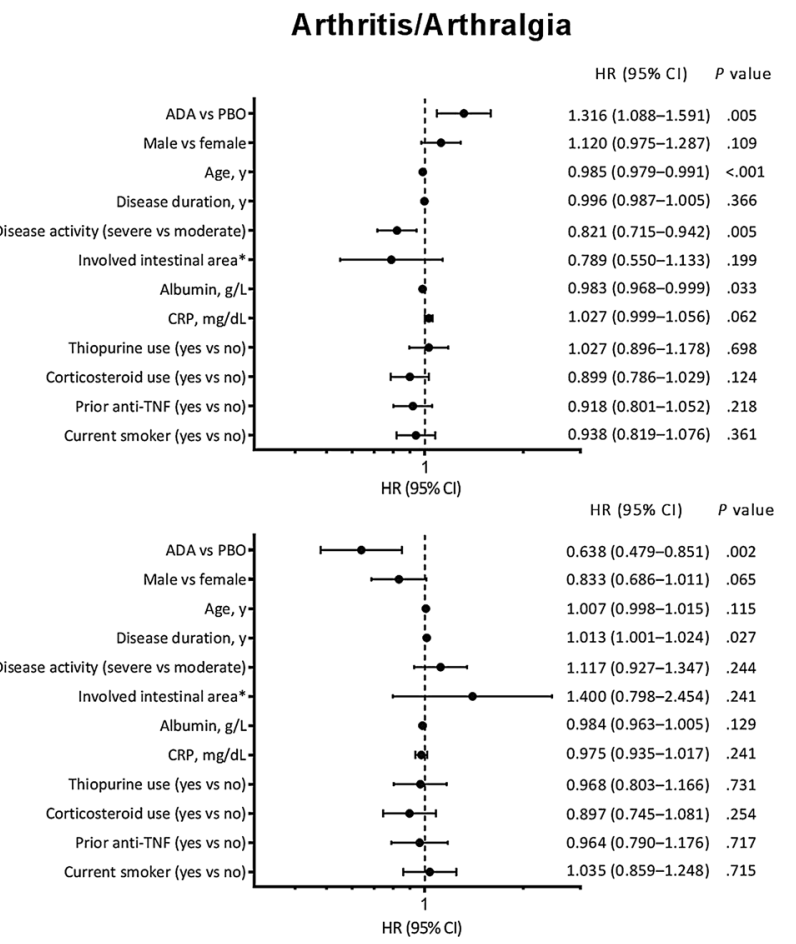

extraintestinal manifestation, $P B O$ placebo, $T N F$ tumor necrosis factor. Asterisk indicates ileal, colonic, gastroduodenal versus other

cohort data. In an open-label study, partial response or remission of peripheral arthritis was reported in $61 \%$ of patients with CD receiving adalimumab [39]. Similar findings were reported in an open-label study of infliximab in which $61 \%$ of patients with CD experienced improvement of arthritis and 46\% achieved complete resolution at 12 weeks [32].

Increased risk of developing EIMs has been reported in patients with CD who smoked, had prolonged disease duration, or were younger at first diagnosis [3, 8, 40]. Some EIMs (e.g., erythema nodosum) are also reported to affect women more commonly than men $[8,27,28,40]$. Our analysis supports these findings and demonstrated that significantly more patients with EIMs at baseline were female, current smokers, and had severe (versus moderate) disease activity compared with patients without EIMs. Patients with EIMs were also more likely to be older and have longer disease duration. Predictors of initial and 
durable EIM resolution included adalimumab treatment, male sex, and moderate (versus severe) disease activity at baseline. We did not find an association between resolution of EIMs and smoking status. Because of the large size of this study, other variables were found to be statistically significant but may not be clinically meaningful.

There were a few limitations in this analysis. First, this was a post hoc analysis of trials with different study designs (including both randomized and open-label trials). Per study designs, the duration of placebo exposure was limited in many trials, leading to an imbalanced number of evaluable patients at 6 months and 1 year. In addition, given that only a small proportion of patients had ocular or cutaneous EIMs at baseline, it was difficult to evaluate the full contribution of adalimumab to resolution of these EIMs. Finally, in the studies that used the CDAI, we were able to analyze the general category of EIMs (e.g., ocular or cutaneous) but not specific EIMs within each category. Consequently, inclusion of arthralgia, which is common in the general population, may have overestimated the efficacy reported in the arthritis/arthralgia category versus assessing arthritis alone. Furthermore, as a result of the way EIMs were captured in the clinical trials assessed, it was not possible to differentiate between arthropathies that are independent or dependent of disease activity.

\section{CONCLUSION}

This pooled analysis of more than 2000 patients with moderate to severe $\mathrm{CD}$ demonstrated that treatment with adalimumab was effective in initial and durable resolution of any EIM and arthritis and/or arthralgia.

\section{ACKNOWLEDGEMENTS}

Funding. Sponsorship for this study, article processing charges, and the open access fee were funded by AbbVie (North Chicago, IL, USA).
AbbVie reviewed and approved the manuscript for submission.

Medical Writing and Editorial Assistance. Medical writing support was provided by Maria Hovenden, PhD, at Complete Publication Solutions, LLC (North Wales, PA, USA), a CHC Group company, and was funded by AbbVie.

Authorship. All named authors meet the International Committee of Medical Journal Editors (ICMJE) criteria for authorship for this manuscript, take responsibility for the integrity of the work as a whole, and have given final approval for the version to be published. All authors had full access to all of the data in this study and take complete responsibility for the integrity of the data and accuracy of the data analysis.

Disclosures. Edouard J. Louis has received fees for educational Grants from MSD, AbbVie, and Takeda; speakers fees from AbbVie, Ferring, MSD, Chiesi, Mitsubishi Pharma, Hospira, Janssen, and Takeda; and advisory board fees from AbbVie, Ferring, MSD, Takeda, Hospira, Mitsubishi Pharma, Celltrion, and Prometheus. Walter Reinisch has served as a speaker for Abbott Laboratories, AbbVie, Aesca, Aptalis, Astellas, Centocor, Celltrion, Danone Austria, Elan, Falk Pharma GmbH, Ferring, Immundiagnostik, Mitsubishi Tanabe Pharma Corporation, MSD, Otsuka, PDL, Pharmacosmos, PLS Education, Schering-Plough, Shire, Takeda, Therakos, Vifor, and Yakult; as a consultant for Abbott Laboratories, AbbVie, Aesca, Amgen, AM Pharma, Astellas, AstraZeneca, Avaxia, Roland Berger GmBH, Bioclinica, Biogen IDEC, Boehringer-Ingelheim, Bristol-Myers Squibb, Cellerix, Chemocentryx, Celgene, Centocor, Celltrion, Covance, Danone Austria, Elan, Ernst \& Young, Falk Pharma GmbH, Ferring, Galapagos, Genentech, Gilead, Grünenthal, ICON, Index Pharma, Inova, Janssen, Johnson \& Johnson, Kyowa Hakko Kirin Pharma, Lipid Therapeutics, Mallinckrodt, MedImmune, Millenium, Mitsubishi Tanabe Pharma Corporation, MSD, Nestle, Novartis, Ocera, Otsuka, Parexel, PDL, Pharmacosmos, Pfizer, Procter \& Gamble, Prometheus, Robarts Clinical Trial, 
Schering-Plough, Second Genome, Setpointmedical, Sigmoid, Takeda, Therakos, Tigenix, UCB, Vifor, Zealand, Zyngenia, and 4SC; as an advisory board member for Abbott Laboratories, AbbVie, Aesca, Amgen, AM Pharma, Astellas, AstraZeneca, Avaxia, Biogen IDEC, Boehringer-Ingelheim, Bristol-Myers Squibb, Cellerix, Chemocentryx, Celgene, Centocor, Celltrion, Danone Austria, Elan, Ferring, Galapagos, Genentech, Grünenthal, Inova, Janssen, Johnson \& Johnson, Kyowa Hakko Kirin Pharma, Lipid Therapeutics, MedImmune, Millenium, Mitsubishi Tanabe Pharma Corporation, MSD, Nestle, Novartis, Ocera, Otsuka, PDL, Pharmacosmos, Pfizer, Procter \& Gamble, Prometheus, Schering-Plough, Second Genome, Setpointmedical, Takeda, Therakos, Tigenix, UCB, Zealand, Zyngenia, and 4SC; and has received research funding from Abbott Laboratories, AbbVie, Aesca, Centocor, Falk Pharma GmbH, Immundiagnostik, and MSD. David A. Schwartz has received Grant support from AbbVie and UCB and consultancy fees from AbbVie, UCB, Janssen, Takeda, and Tigenix. Robert Löfberg has received research Grants from Sophiahemmet and the Stichting af Jochnick Foundation; lecture fees from Abbott, Asahi-Kasei, AstraZeneca, Celltech, Centocor, Connexion, Cosmo, Elan, InDex Pharmaceuticals, Meda, Otsuka, Pharmacia-Pfizer, ScheringPlough, Schering AG, Serono, and UCB; and consultancy fees from Abbott, Asahi-Kasei, AstraZeneca, Celltech, Centocor, Connexion, Cosmo, Elan, InDex Pharmaceuticals, Meda, Otsuka, Pharmacia-Pfizer, Schering-Plough, Schering AG, Serono, and UCB. Anne M. Robinson is an AbbVie employee and may own AbbVie stock and/or options. Sofie Berg is an AbbVie employee and may own AbbVie stock and/or options. Anthony W. Wang is an AbbVie employee and may own AbbVie stock and/or options. Jen-fue Maa is an AbbVie employee and may own AbbVie stock and/or options. Bidan Huang is an AbbVie employee and may own AbbVie stock and/or options. Brandee Pappalardo is an AbbVie employee and may own AbbVie stock and/or options.

Compliance with Ethics Guidelines. This article does not contain any new studies with human or animal subjects performed by any of the authors.

Data Availability. The datasets generated during and/or analyzed during the current study are available on request through the "Clinical Trials Data \& Information Sharing" portal (https://www.abbvie.com/our-science/ clinical-trials/clinical-trials-data-and-

information-sharing.html).

Open Access. This article is distributed under the terms of the Creative Commons Attribution-NonCommercial 4.0 International License (http://creativecommons.org/licenses/ by-nc/4.0/), which permits any noncommercial use, distribution, and reproduction in any medium, provided you give appropriate credit to the original author(s) and the source, provide a link to the Creative Commons license, and indicate if changes were made.

\section{REFERENCES}

1. Zippi M, Corrado C, Pica R, et al. Extraintestinal manifestations in a large series of Italian inflammatory bowel disease patients. World J Gastroenterol. 2014;20:17463-7.

2. Vavricka SR, Brun L, Ballabeni P, et al. Frequency and risk factors for extraintestinal manifestations in the Swiss inflammatory bowel disease cohort. Am J Gastroenterol. 2011;106:110-9.

3. Ott C, Takses A, Obermeier F, Schnoy E, Muller M. Smoking increases the risk of extraintestinal manifestations in Crohn's disease. World J Gastroenterol. 2014;20:12269-76.

4. Isene R, Bernklev $\mathrm{T}$, Hoie $\mathrm{O}$, et al. Extraintestinal manifestations in Crohn's disease and ulcerative colitis: results from a prospective, population-based European inception cohort. Scand J Gastroenterol. 2015;50:300-5.

5. Harbord M, Annese V, Vavricka SR, et al. The first European evidence-based consensus on extra-intestinal manifestations in inflammatory bowel disease. J Crohns Colitis. 2016;10:239-54.

6. Williams H, Walker D, Orchard TR. Extraintestinal manifestations of inflammatory bowel disease. Curr Gastroenterol Rep. 2008;10:597-605. 
7. Ardizzone S, Puttini PS, Cassinotti A, Porro GB. Extraintestinal manifestations of inflammatory bowel disease. Dig Liver Dis. 2008;40:S253-9.

8. Roberts H, Rai SN, Pan J, et al. Extraintestinal manifestations of inflammatory bowel disease and the influence of smoking. Digestion. 2014;90:122-9.

9. Vavricka SR, Scharl M, Gubler M, Rogler G. Biologics for extraintestinal manifestations of IBD. Curr Drug Targets. 2014;15:1064-73.

10. Peyrin-Biroulet L, Van Assche G, Gomez-Ulloa D, et al. Systematic review of tumor necrosis factor antagonists in extraintestinal manifestations in inflammatory bowel disease. Clin Gastroenterol Hepatol. 2017;15(25-36):e27.

11. Sandborn WJ, Hanauer SB, Rutgeerts P, et al. Adalimumab for maintenance treatment of Crohn's disease: results of the CLASSIC II trial. Gut. 2007;56:1232-9.

12. Colombel JF, Sandborn WJ, Rutgeerts P, et al. Adalimumab for maintenance of clinical response and remission in patients with Crohn's disease: the CHARM trial. Gastroenterology. 2007;132:52-65.

13. Hanauer SB, Sandborn WJ, Rutgeerts $P$, et al. Human anti-tumor necrosis factor monoclonal antibody (adalimumab) in Crohn's disease: the CLASSIC-I trial. Gastroenterology. 2006;130:323-33.

14. Hyams JS, Griffiths A, Markowitz J, et al. Safety and efficacy of adalimumab for moderate to severe Crohn's disease in children. Gastroenterology. 2012;143:365-74.

15. Sandborn WJ, Rutgeerts P, Enns R, et al. Adalimumab induction therapy for Crohn disease previously treated with infliximab: a randomized trial. Ann Intern Med. 2007;146:829-38.

16. Panaccione R, Colombel JF, Sandborn WJ, et al. Adalimumab sustains clinical remission and overall clinical benefit after 2 years of therapy for Crohn's disease. Aliment Pharmacol Ther. 2010;31:1296-309.

17. Rutgeerts P, Van Assche G, Sandborn WJ, et al. Adalimumab induces and maintains mucosal healing in patients with Crohn's disease: data from the EXTEND trial. Gastroenterology. 2012;142:1102-11.

18. Lofberg R, Louis EV, Reinisch W, et al. Adalimumab produces clinical remission and reduces extraintestinal manifestations in Crohn's disease: results from CARE. Inflamm Bowel Dis. 2012;18:1-9.

19. Watanabe M, Hibi T, Lomax KG, et al. Adalimumab for the induction and maintenance of clinical remission in Japanese patients with Crohn's disease. J Crohns Colitis. 2012;6:160-73.
20. Panaccione R, Colombel JF, Sandborn WJ, et al. Adalimumab maintains remission of Crohn's disease after up to 4 years of treatment: data from CHARM and ADHERE. Aliment Pharmacol Ther. 2013;38:1236-47.

21. Panaccione R, Sandborn WJ, D'Haens GR, et al. Adalimumab maintains long-term remission in Crohn's disease after infliximab failure: 1-year follow-up of GAIN trial. J Crohns Colitis. 2008;134:A133-4.

22. Faubion WA, Dubinsky $M$, Ruemmele FM, et al. Long-term efficacy and safety of adalimumab in pediatric patients with Crohn's disease. Inflamm Bowel Dis. 2017;23:453-60.

23. Smale S, Natt RS, Orchard TR, Russell AS, Bjarnason I. Inflammatory bowel disease and spondylarthropathy. Arthritis Rheum. 2001;44:2728-36.

24. Gravallese EM, Kantrowitz FG. Arthritic manifestations of inflammatory bowel disease. Am J Gastroenterol. 1988;83:703-9.

25. Peyrin-Biroulet L, Cieza A, Sandborn WJ, et al. Development of the first disability index for inflammatory bowel disease based on the international classification of functioning, disability and health. Gut. 2012;61:241-7.

26. Vavricka SR, Rogler G, Gantenbein C, et al. Chronological order of appearance of extraintestinal manifestations relative to the time of IBD diagnosis in the Swiss Inflammatory Bowel Disease Cohort. Inflamm Bowel Dis. 2015;21:1794-800.

27. Orchard TR, Chua CN, Ahmad T, Cheng H, Welsh KI, Jewell DP. Uveitis and erythema nodosum in inflammatory bowel disease: clinical features and the role of HLA genes. Gastroenterology. 2002;123:714-8.

28. Bernstein $\mathrm{CN}$, Blanchard JF, Rawsthorne $\mathrm{P}, \mathrm{Yu} \mathrm{N}$. The prevalence of extraintestinal diseases in inflammatory bowel disease: a population-based study. Am J Gastroenterol. 2001;96:1116-22.

29. Larsen S, Bendtzen K, Nielsen OH. Extraintestinal manifestations of inflammatory bowel disease: epidemiology, diagnosis, and management. Ann Med. 2010;42:97-114.

30. van Sommeren S, Janse M, Karjalainen J, et al. Extraintestinal manifestations and complications in inflammatory bowel disease: from shared genetics to shared biological pathways. Inflamm Bowel Dis. 2014;20:987-94.

31. Rubin D, Feagan B, Dryden G, et al. The effect of vedolizumab on extraintestinal manifestations in 
patients with Crohn's disease in GEMINI 2 . Inflamm Bowel Dis. 2016;22:S42-3.

32. Herfarth H, Obermeier F, Andus T, et al. Improvement of arthritis and arthralgia after treatment with infliximab (Remicade) in a German prospective, open-label, multicenter trial in refractory Crohn's disease. Am J Gastroenterol. 2002;97:2688-90.

33. Barrie A, Regueiro M. Biologic therapy in the management of extraintestinal manifestations of inflammatory bowel disease. Inflamm Bowel Dis. 2007;13:1424-9.

34. Generini S, Giacomelli R, Fedi R, et al. Infliximab in spondyloarthropathy associated with Crohn's disease: an open study on the efficacy of inducing and maintaining remission of musculoskeletal and gut manifestations. Ann Rheum Dis. 2004;63:1664-9.

35. Ellman MH, Hanauer S, Sitrin M, Cohen R. Crohn's disease arthritis treated with infliximab: an open trial in four patients. J Clin Rheumatol. 2001;7:67-71.

36. Regueiro M, Valentine J, Plevy S, Fleisher MR, Lichtenstein GR. Infliximab for treatment of pyoderma gangrenosum associated with inflammatory bowel disease. Am J Gastroenterol. 2003;98:1821-6.

37. Rudwaleit M, Rodevand E, Holck P, et al. Adalimumab effectively reduces the rate of anterior uveitis flares in patients with active ankylosing spondylitis: results of a prospective open-label study. Ann Rheum Dis. 2009;68:696-701.

38. Murphy CC, Ayliffe WH, Booth A, Makanjuola D, Andrews PA, Jayne D. Tumor necrosis factor alpha blockade with infliximab for refractory uveitis and scleritis. Ophthalmology. 2004;111:352-6.

39. Barreiro-de-Acosta M, Lorenzo A, DominguezMunoz JE. Efficacy of adalimumab for the treatment of extraintestinal manifestations of Crohn's disease. Rev Esp Enferm Dig. 2012;104:468-72.

40. Karmiris K, Avgerinos A, Tavernaraki A, et al. Prevalence and characteristics of extra-intestinal manifestations in a large cohort of Greek patients with inflammatory bowel disease. J Crohns Colitis. 2016;10:429-36. 